\title{
Picosecond time-resolved study of excitons in GaAs-AlAs multi-quantum-well structures
}

\author{
Yasuaki Masumoto and Shigeo Shionoya \\ The Institute for Solid State Physics, The University of Tokyo, Roppongi 7-22-1, Minato-ku, \\ Tokyo 106, Japan \\ Hitoshi Kawaguchi \\ Musashino Electrical Communication Laboratory, Nippon Telegraph and Telephone Public Corporation (NTT), \\ Midori-machi 3-9-11, Musashino-shi, Tokyo 180, Japan
}

(Received 1 December 1983)

\begin{abstract}
Energy- and time-resolved measurements of luminescence of $1 s$ excitons $(n=1, e-h h)$ in GaAs-AlAs multi-quantum-well structures have been carried out for the first time in the picosecond time domain. Dynamical population changes of excitons are directly visualized in the energy-time coordinates. Results indicate that excitons lose their energy in the exciton band at a rate of $1.0 \times 10^{6} \mathrm{eV} / \mathrm{s}$. This rate is much slower than the calculated kinetic-energy-loss rate and is ascribed to the random nature of the well.
\end{abstract}

Excitons in multi-quantum-well (MQW) structures have prominent two-dimensional characters because of the quantum size effect. ${ }^{1}$ Wave functions and binding energies of excitons in MQW are quite different from those of excitons in bulk crystals as a result of this effect. ${ }^{2-4}$ In addition to the two-dimensional characters, excitons in MQW have an inhomogeneous character which is inevitably given in the process of the sample preparation. ${ }^{5,6}$ The well thickness is considered to be fluctuated by an order of one atomic layer. This yields the fluctuation of the resonance energy of excitons. Thus excitons in MQW are considered as a typical example of the two-dimensional excitons in the random potential.

Energetical relaxation processes of excitons in the twodimensional random well are expected to be quite different from those of excitons in three-dimensional regular crystals. However, to our knowledge, there exist no experimental as well as theoretical studies dealing with energetical relaxation processes of excitons in the two-dimensional random well. In this work, the energy- and time-resolved luminescence of weakly excited excitons in MQW has been studied for the first time. Energetical relaxation processes of excitons in MQW are discussed on the basis of the experimental results and of a simple calculation which takes account of the twodimensional character of excitons.

The sample used in this experiment was grown by molecular beam epitaxy (MBE) on a GaAs (100) substrate. It consists of alternate $76-\AA \mathrm{GaAs}$ well layers and $33-\AA$ AlAs barrier layers of the total thickness $2.98 \mu \mathrm{m}$. A window was etched in the GaAs substrate for the optical absorption measurement.

A rhodamine 6-G dye laser synchronously pumped by a mode-locked argon laser was used, which gives 1-2-ps light pulses of $300 \mathrm{pJ}$. The pulse duration was continually monitored by using a rapid-scan autocorrelator. ${ }^{7}$ The lasing photon energy was $2.063 \mathrm{eV}$ with a spectral width of $3 \mathrm{meV}$. The laser beam was focused on a GaAs-AlAs MQW sample immersed in liquid helium with a spot size of $200 \mu \mathrm{m}$. The spectrally resolved temporal response of the luminescence was analyzed by using a combined system of a $25-\mathrm{cm}$ monochromator, a synchroscan streak camera (HTV-C1587), an SIT (silicon intensifier target) camera, and a computer. The streak camera was synchronously operated with the modelocked argon laser. The averaging at a high repetition rate (82 $\mathrm{MHz}$ ) enabled us to observe directly the spectrally resolved [full width at half maximum (FWHM) $=1.1$ $\mathrm{meV}$ ] temporal response of the exciton luminescence from GaAs-AlAs MQW excited by only 300-pJ light pulses. Time resolution of the streak camera was found to be 30 ps. However, the time resolution of the combined system of the monochromator and the streak camera was lowered to $70 \mathrm{ps}$, because of the spread of the light path in the monochromator.

In Fig. 1, spectra of the absorption and time-integrated luminescence are shown. The absorption peak observed at $1.6225 \mathrm{eV}$ with 7.5-meV width (FWHM) is ascribed to the $1 s$ exciton $(n=1, e-h h)$ composed of an electron and a heavy hole belonging to the lowest state $(n=1)$ in the quantum well. The observed spectral width is attributed to

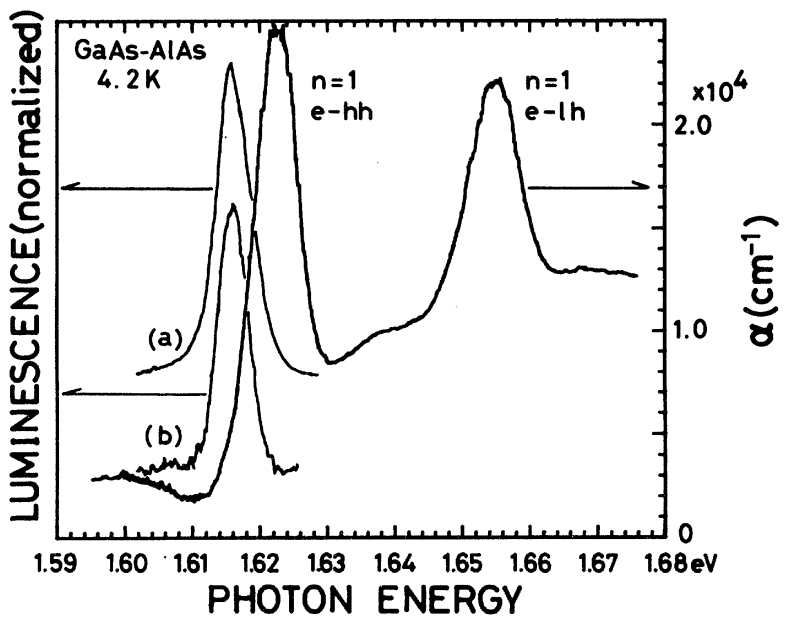

FIG. 1. Absorption coefficient and time-integrated luminescence spectra of GaAs-AlAs MQW (76- $\AA$ GaAs well, 33- $\AA$ AlAs barrier, $2.98 \mu \mathrm{m})$ at $4.2 \mathrm{~K}$. The spectral resolution is $0.3 \mathrm{meV}$. Luminescence spectra were obtained under (a) pulsed dye laser (2.063 eV, 1-2 ps, $300 \mathrm{pJ})$ and (b) $\mathrm{cw} \mathrm{He}-\mathrm{Ne}$ laser (1.959 eV, 0.01 $\mathrm{mW}$ ) excitation. 
the fluctuation of the well thickness from the following discussion. ${ }^{5}$ The energy of the $1 s$ exciton in the well measured from the bottom of the well (the band-gap energy of bulk GaAs) is $E_{n-1}(1 s)=1.6225 \mathrm{eV}-E_{g}=103.3 \mathrm{meV}$, where $E_{\mathrm{g}}=1.5192 \mathrm{eV}$ is the band-gap energy of GaAs. ${ }^{8}$ Assuming the fluctuation of the well width to be the half of the lattice constant $a$ of GaAs and noting that $E_{n-1}(1 s)$ is approximately proportional to the inverse square of the well thickness $L_{z},{ }^{2,3}$ one can calculate the fluctuation of $E_{n-1}(1 s)$ in MQW as

$$
\begin{aligned}
\delta E_{n-1}(1 s) & \cong 2 E_{n=1}(1 s)\left(\delta L_{z} / L_{z}\right) \\
& =2 E_{n=1}(1 s)\left(a / 2 L_{z}\right)=7.7 \mathrm{meV} .
\end{aligned}
$$

Here $\delta E_{n-1}(1 s)$ is the fluctuation of $E_{n=1}(1 s), L_{z}=76 \AA$ is the well thickness, $\delta L_{z}$ is the fluctuation of $L_{z}$, and $a=5.64$ $\AA .{ }^{9}$ Because this estimated value of $7.7 \mathrm{meV}$ almost coincides with the measured value of $7.5 \mathrm{meV}$, it is concluded that the width of absorption arises from the fluctuation of the well width which is $\sim a / 2 .{ }^{10}$

A time-integrated luminescence band under the 1-2-ps pulse excitation is observed at the lower-energy side of the absorption band as shown in Fig. 1. Peaks of the luminescence and absorption spectra are separated by $6.5 \mathrm{meV}$. This fact indicates that photogenerated excitons are populated at the low-energy states in the exciton band, because the luminescence spectra reflect the energetical distribution of excitons. The excitation photon energy $(=2.063 \mathrm{eV})$ in the present case is above the band-gap energy of GaAs and below the indirect $(=2.229 \mathrm{eV} ; X)$ as well as direct $(=3.13$ $\mathrm{eV} ; \Gamma)$ excitonic band-gap energy of AlAs. ${ }^{11}$ Therefore, only the GaAs well layers are photoexcited. The number of photoexcited layers is less than 20 , because the absorption coefficient for $2.063 \mathrm{eV}$ is above the detection limit $\left(5 \times 10^{4}\right.$ $\left.\mathrm{cm}^{-1}\right)$. The luminescence band changes little, even when the excitation level is reduced to $0.01 \mathrm{~mW}$ (CW) $\mathrm{He}-\mathrm{Ne}$ laser excitation. Therefore, it is sure that the luminescence band observed under the $1 \sim 2$-ps $(300 \mathrm{pJ})$ pulse excitation arises from $1 s$ excitons. Any other luminescence bands, such as those due to $2 s$ excitons and $1 s$ excitons $(n=1, e-l h)$ in MQW, the AlAs barrier layers, and the GaAs substrate, were not observed.

The energy- and time-resolved results of luminescence are shown in Figs. 2 and 3. These figures are constructed from the spectrally resolved temporal response of luminescence. As is seen from the figures, the observed rise of the population of excitons is determined by the time resolution of the instrument at the high-energy extremity $(1.6228 \mathrm{eV})$. On the other hand, it takes about 400 ps for the population of excitons lower than $1.616 \mathrm{eV}$ to reach its maximum. Because the reflectivity in the relevant energy range was found not to change very much $(\Delta R / R<0.2)$, the change of the refractive index also should be small. Thus the energy and time responses of luminescence can be regarded as directly reflecting the dynamics of the exciton population, because the energy dependence of the emission probability of excitons can be neglected. The temporal change of population is clearly observed in Fig. 3. Excitons drift toward lowerenergy states losing their energy. The spectrally integrated luminescence does not show a single exponential decay. However, a characteristic time to decay to the $1 / e$ value of the maximum is about 480 ps. This decay characteristic does not depend on the excitation intensity down to $10 \%$.

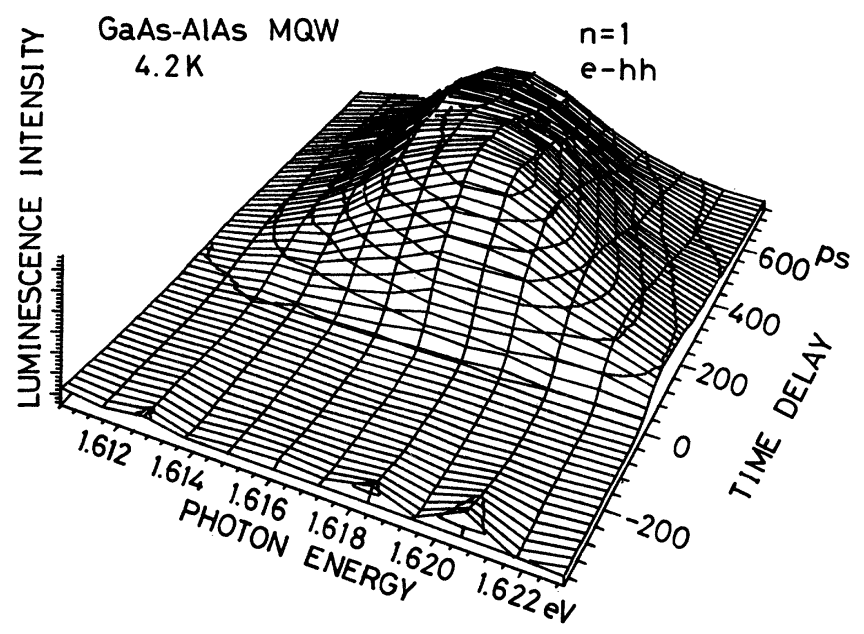

FIG. 2. A bird's-eye view of energy- and time-resolved luminescence intensity of the excitons $(n=1, e-h h)$ in GaAs-AlAs MQW at $4.2 \mathrm{~K}$.

Therefore, we need not take account of the effect of the bimolecular recombination.

To discuss the energetical relaxation of excitons, the average energy $\langle E(t)\rangle$ of the ensemble of excitons is defined as follows:

$$
\langle E(t)\rangle=\left(\sum_{i=1}^{12} E_{i} f\left(E_{i}, t\right)\right) /\left(\sum_{i=1}^{12} f\left(E_{i}, t\right)\right),
$$

where $f\left(E_{i}, t\right)$ is the spectrally resolved temporal response of the luminescence shown in Fig. 2, and $E_{i}$ 's $(i=1-12)$ correspond to the observed photon energies. The temporal change of $\langle E(t)\rangle$ is shown in Fig. 4. The result clearly shows that the average energy of excitons decreases at a constant rate of $1.0 \times 10^{6} \mathrm{eV} / \mathrm{s}$.

The kinetic energy decreasing rate of two-dimensional excitons via the deformation-potential-type interaction with LA phonons is calculated by the following equation, which is a direct extension of the expression describing the energy decreasing rate of two-dimensional hot electrons, ${ }^{12-14}$

$$
\langle d E(t) / d t\rangle_{\mathrm{dp}}=-\left(2 M^{2} D^{2} / \hbar^{3} \rho\right)\left[k_{B} T_{e}(t)-k_{B} T_{L}\right],
$$

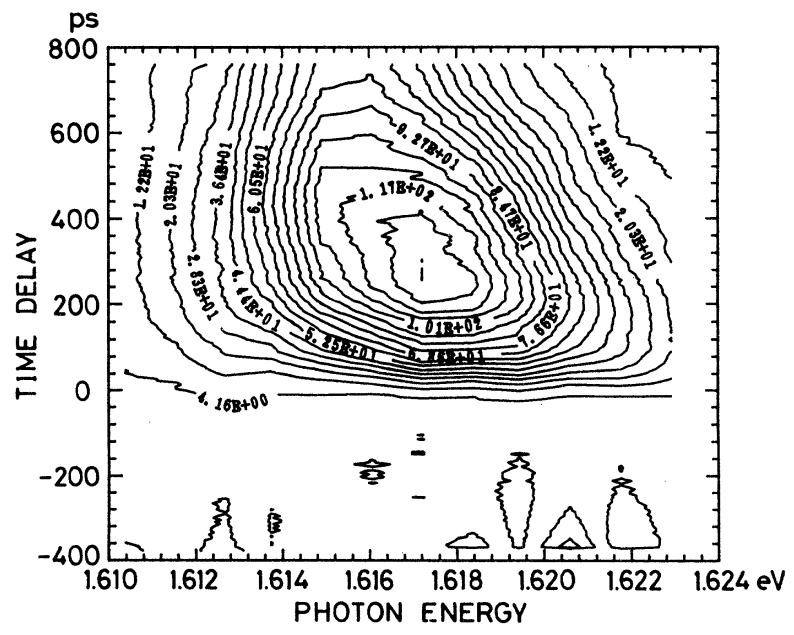

FIG. 3. A contour map of Fig. 2. 


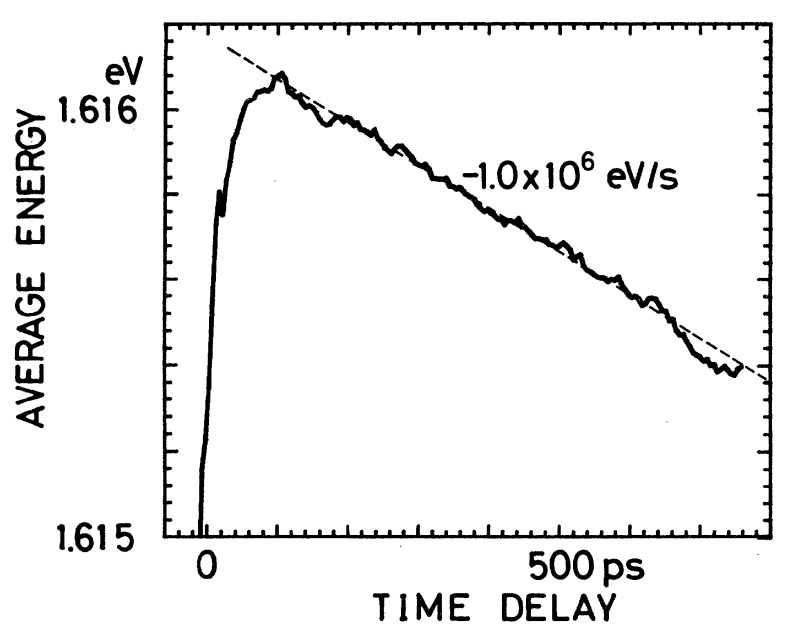

FIG. 4. Temporal change of the average energy of the excitons $(n=1, e-h h)$ in GaAs-AlAs MQW at $4.2 \mathrm{~K}$.

where $M$ is the exciton translational mass, $D$ is the exciton deformation potential, $\rho$ is the areal mass density in a layer thickness $L_{z}, T_{e}(t)$ is the effective temperature of twodimensional excitons, and $T_{L}$ is the lattice temperature. The piezoelectric-type interaction with LA or TA phonons is considered to give the minor contribution to the energydecreasing rate of two-dimensional excitons compared with the deformation-potential-type interaction in GaAs except at the large wave vector of excitons. ${ }^{15,16}$ The Fröhlich-type interaction with LO phonons also does not contribute to the energy relaxation of excitons in the exciton band, because LO phonon energy $(=36.2 \mathrm{meV})$ is larger than the observed luminescence bandwidth. Equation (3) is integrated as follows, because $\langle E(t)\rangle=k_{B} T_{e}(t)$ :

$T_{e}(t)=T_{L}+\left[T_{e}(0)-T_{L}\right] \exp \left[-\left(2 M^{2} D^{2} / \hbar^{3} \rho\right) t\right]$,

where $T_{e}(0)$ is the initial temperature of the excitons. Using the values of $M=0.7 m_{0}$ (electron mass), ${ }^{17} D=9.6 \mathrm{eV},{ }^{18}$ $\rho=\rho_{3-\operatorname{dim}} L_{z}=\left(5.3 \mathrm{~g} / \mathrm{cm}^{3}\right) \times\left(76 \times 10^{-8} \mathrm{~cm}\right)=4.0 \times 10^{-6}$ $\mathrm{g} / \mathrm{cm}^{2},{ }^{9}$ and $T_{L}=4.2 \mathrm{~K}$, the exponent in Eq. (4) is obtained as $-\left(2 M^{2} D^{2} / \hbar^{3} \rho\right) t=-\left(4.1 \times 10^{10} \mathrm{~s}^{-1}\right) t$. This indicates that two-dimensional excitons lose their kinetic energy at a time constant of 24 ps. This calculated decay rate is much faster than the observed one, if all the energy losses are attributed to the kinetic-energy loss.

In the above calculation, we have taken account only of the two dimensionality of excitons. In reality, not only the two dimensionality but also the randomness due to the fluctuation of the well width contributes to the energetical relaxation of excitons. In MQW there should be a number of clusters in which the well width is homogeneous. The lateral characteristic size $1_{c}$ of the clusters is estimated to be an order of the exciton Bohr radius $a_{B}=136 \AA$, ${ }^{19}$ because the absorption spectrum of excitons does not show substructures. Because the excitation spot size of $200 \mu \mathrm{m}$ covers many (an order of $10^{8}$ ) clusters, the statistical distribution of the lateral size of clusters in each photoexcited well structure is expected to be the same. Supposing the extreme case that the cluster lateral size is much larger than $a_{B}$, the exciton energy levels in each cluster are different from each other by the multiple of $7.7 \mathrm{meV}$ in our sample, because the fluctuation of the well width is the multiple of $a / 2$. Around the cluster boundaries, the intercluster translational motion of excitons is restricted, because the mismatch of the resonance energy of $1 s$ excitons should be compensated by the emission or absorption of acoustic phonons. Thus excitons are localized in clusters with the loss of the kinetic energy and intercluster movement of excitons is weakly allowed with the emission or absorption of acoustic phonons.

Similarly to the three-dimensional case, photogenerated two-dimensional electron-hole pairs will quickly lose their energy to form excitons with the emission of a number of phonons within the present time resolution. ${ }^{20}$ The calculation described in Eq. (4) shows that the kinetic energy loss of excitons is almost completed within the present time resolution. What process is responsible for the observed energy-loss rate of excitons? In the random well, there can be two channels for the energetic relaxation processes of excitons. On the one hand, excitons lose their kinetic energy via the acoustic-phonon interaction. On the other hand, excitons lose their energy migrating toward the lower-energy positions in an inhomogeneous well with the emission of acoustic phonons. After most of the kinetic energy is lost, the kinetic-energy-loss process works little because $\langle d E(t) / d t\rangle_{\mathrm{dp}}$ is proportional to the excess kinetic energy $k_{B} T_{e}(t)-k_{B} T_{L}$, so that excitons lose their energy mainly via the latter process. If this is correct, the estimation based on Eq. (3) and the result of Fig. 4 show that the alternation of the energy-loss channels occurs when the excess kinetic energy of excitons becomes smaller than $0.024 \mathrm{meV}$. Then the localization of the excitons is established with the loss of the kinetic energy. After that, excitons lose their energy slowly, migrating toward lower-energy positions in the inhomogeneous well with the emission of acoustic phonons. As stated above, the intercluster migration of excitons presumably interprets the observed slow energy-decreasing rate of excitons, although at present we cannot calculate quantitatively the energy-decreasing rate on the basis of this model. Detailed experiments on various samples having different degrees of two dimensionality and randomness are now in progress.

In summary, picosecond relaxation processes of $1 \mathrm{~s}$ excitons $(n=1, e-h h)$ in GaAs-AlAs MQW have been studied by observing the energy- and time-resolved luminescence. The decreasing rate of the average energy of excitons is constant and found to be $1.0 \times 10^{6} \mathrm{eV} / \mathrm{s}$. This rate is much slower than the kinetic-energy-loss rate calculated by taking account of the two dimensionality of excitons. Instead, the energetic relaxation process due to intercluster migration of excitons with the emission of acoustic phonons is proposed to interpret the observed slow rate.

\section{ACKNOWLEDGMENTS}

The authors wish to deeply thank Dr. H. Okamoto, Dr. Y. Horikoshi, and Dr. S. Tarucha at Musashino Electrical Communication Laboratory (Nippon Telegraph and Telephone Public Corporation) for providing high-quality samples and for valuable discussions. They thank Professor $\mathbf{M}$. Matsuura at Yamaguchi University for sending them a report of work prior to publication. They also thank Dr. T. Takagahara at the University of Tokyo for valuable discussions and the critical reading of the manuscript. 
${ }^{1} \mathbf{R}$. Dingle, in Festkörperprobleme $X V$, edited by H. J. Queisser (Pergamon, New York, 1975), p. 21.

${ }^{2}$ G. Bastard, E. E. Mendez, L. L. Chang, and L. Esaki, Phys. Rev. B 26, 1974 (1982).

${ }^{3}$ Y. Shinozuka and M. Matsuura, Phys. Rev. B 28, 4878 (1983).

${ }^{4}$ T. Ishibashi, S. Tarucha, and H. Okamoto, in Gallium Arsenide and Related Compounds, 1981, edited by T. Sugano (The Institute of Physics, London, 1982), p. 587.

${ }^{5}$ C. Weisbuch, R. Dingle, A. C. Gossard, and W. Wiegmann, Solid State Commun. 38, 709 (1981).

${ }^{6} \mathrm{~L}$. Goldstein, Y. Horikoshi, S. Tarucha, and H. Okamoto, Jpn. J. Appl. Phys. 22, 1489 (1983).

${ }^{7}$ Y. Ishida, T. Yajima, and Y. Tanaka, Jpn. J. Appl. Phys. 19, L289 (1980).

${ }^{8}$ D. D. Sell, Phys. Rev. B $\underline{6}, 3750$ (1972).

${ }^{9} \mathrm{M}$. Neuberger, in III-V Semiconducting Compounds, Handbook of Electronic Materials, edited by $M$. Neuberger, (Plenum, New York, 1971), Vol. 2, p. 45.

${ }^{10}$ The observed resonance energy of $1 s$ excitons $(n=1, e-h h)$ deviates from the calculation based on the finite-well model by 22.6 $\mathrm{meV}$, while it coincides with the calculation based on the infinitewell model within an error of $1.4 \mathrm{meV}$. This suggests that excitons are completely confined in the well and that the transfer of the excitons between wells is negligible. Thus the broadening of the level by the tunneling is considered to be negligible. Even if one takes the multi-finite-well model, the calculation shows that the broadenings of the lowest-energy levels of electrons and holes by the tunneling through barriers are 0.56 and $0.039 \mathrm{meV}$, respectively. These values are much smaller than the observed spectral width of $7.5 \mathrm{meV}$.

11B. Monemar, Phys. Rev. B 8 , 5711 (1973).

${ }^{12}$ K. Hess and C. T. Sah, Phys. Rev. B 10,3375 (1974).

${ }^{13}$ Y. Toyozawa, Prog. Theor. Phys. 20, 53 (1958).

${ }^{14} \mathrm{Y}$. Masumoto (unpublished).

${ }^{15} \mathrm{R}$. G. Ulbrich and C. Weisbuch, in Festkorperprobleme XVIII, edited by J. Treusch (Vieweg, Braunschweig, 1978), p. 217.

${ }^{16} \mathrm{C}$. Weisbuch and R. G. Ulbrich, in Light Scattering in Solids III, edited by M. Cardona and G. Güntherodt (Springer, Berlin, 1982), p. 207.

${ }^{17}$ R. G. Ulbrich and C. Weisbuch, Phys. Rev. Lett. $\underline{38}, 865$ (1977).

${ }^{18}$ B. Welber, M. Cardona, C. K. Kim, and S. Rodriguez, Phys. Rev. B 12, 5729 (1975).

${ }^{19}$ D. D. Sell, S, E. Stokowski, R. Dingle, and J. V. DiLorenzo, Phys. Rev. B 7, 4568 (1973).

${ }^{20}$ C. V. Shank, R. L. Fork, R. Yen, J. Shah, B. I. Greene, A. C. Gossard, and C. Weisbuch, Solid State Commun. 47, 981 (1983). 